\title{
TITLE:
}

\section{Cryogenic neutron detector comprising an InSb semiconductor detector and a supercritical helium- 3 gas converter}

\section{$\operatorname{AUTHOR}(S)$ :}

Nakamura, Tatsuya; Katagiri, Masaki; Aratono, Yasuyuki; Kanno, Ikuo; Hishiki, Shigeomi; Sugiura, Osamu; Murase, Yasuhiro

\section{CITATION:}

Nakamura, Tatsuya ... [et al]. Cryogenic neutron detector comprising an InSb semiconductor detector and a supercritical helium-3 gas converter. REVIEW OF SCIENTIFIC INSTRUMENTS 2004, 75(2): 340-344

\section{ISSUE DATE:}

2004-02

URL:

http://hdl.handle.net/2433/39809

\section{RIGHT:}

Copyright 2004 American Institute of Physics. This article may be downloaded for personal use only. Any other use requires prior permission of the author and the American Institute of Physics. 


\title{
Cryogenic neutron detector comprising an InSb semiconductor detector and a supercritical helium-3 gas converter
}

\author{
Tatsuya Nakamura, ${ }^{a)}$ Masaki Katagiri, and Yasuyuki Aratono \\ Neutron Science Research Center, Japan Atomic Energy Research Institute, Tokai, Ibaraki 319-1195, Japan \\ Ikuo Kanno and Shigeomi Hishiki \\ Graduate School of Engineering, Kyoto University, Sakyo, Kyoto 606-8501, Japan
}

Osamu Sugiura and Yasuhiro Murase

Graduate School of Science and Engineering, Tokyo Institute of Technology, Meguro, Tokyo 152-8552, Japan

(Received 19 June 2003; accepted 24 November 2003)

\begin{abstract}
We evaluated the neutron-detection characteristics of a proposed cryogenic neutron detector comprising an InSb semiconductor detector and a helium-3 gas converter. The neutron detector was operated at $4.2 \mathrm{~K}$ with helium-3 gas filling up to $1.5 \mathrm{~atm}$, at which the density of the helium-3 nucleus corresponds to that at $160 \mathrm{~atm}$ at room temperature. The secondary particles generated by the ${ }^{3} \mathrm{He}(n, p) \mathrm{T}$ reaction were successfully detected by the InSb detector with a time response of $\sim 80 \mathrm{~ns}$ at all tested gas pressures. (C) 2004 American Institute of Physics.
\end{abstract}

[DOI: $10.1063 / 1.1642747$ ]

\section{INTRODUCTION}

A cryogenic helium-3 neutron detector that can produce precise images of scattered and/or transmitted neutrons of a cooled sample would be very useful, since the detector could be set and cooled very near the sample without any thick obstructions between the source and detector. Implementing this requires a neutron detector that can operate at cryogenic temperatures less than $4.2 \mathrm{~K}$, and exhibit a position resolution of a few tenths of microns and moderate detection efficiency.

Several types of cryogenic neutron detectors have been developed so far, one of which is the cryogenic gas proportional counter. ${ }^{1}$ In that study the gas used was helium-4, but replacing it with helium-3 would result in a cryogenic neutron counter. The authors demonstrated the operation of the gas counter at $5 \mathrm{~K}, 0.013 \mathrm{~atm}$ gas filling, and a gas gain of $\sim 200$. However, much higher gas gain and filling-gas pressure are required to realize a high position resolution. Another type of detector combined a cryogenic solid-state detector and a converter coating film. Baker et al. recently reported that a silicon detector evaporated with lithium fluoride operated well at $80 \mathrm{mK} .^{2}$ This detector was quite successful as a neutron counter, but is not suitable as an imaging detector where hundreds of pixels would need to have coating films of the same thickness for uniform detection efficiency. Moreover, a silicon semiconductor detector is not suitable for use at low temperature since the mobility of the carriers-electrons and holes-becomes small, and is affected by imperfect charge collection.

Hence, here we propose a cryogenic neutron detector that comprises an InSb semiconductor detector and a supercritical helium-3 gas neutron converter. The detector would

${ }^{\text {a)} E l e c t r o n i c ~ m a i l: ~ t a t s @ s t s p 2 a 0 . t o k a i . j a e r i . g o . j p ~}$ exhibit an excellent position resolution less than $0.1 \mathrm{~mm}$. In principle, the InSb detector detects secondary particles created in the following reaction: ${ }^{3} \mathrm{He}+n \rightarrow p$ $+\mathrm{T}\left({ }^{3} \mathrm{He}(n, p) \mathrm{T}\right)$; i.e., neutron detection. Conventional helium-3 filled neutron detectors at room temperature require several atmospheres of helium-3 for high efficiency, and also require an additional, heavier, gas component to provide stopping power if a position resolution on the order of millimeters is required. For example, in helium-3 at $1 \mathrm{~atm}$, the proton range is on the order of $50 \mathrm{~mm}$, and an atmosphere or so of a gas such as $\mathrm{CF}_{4}$ is required to reduce this range to the $\mathrm{mm}$ size. By using the cryogenic environment, one can achieve a helium nucleus with a high density at a relatively low pressure, and the proton range is well below $0.1 \mathrm{~mm}$; for example, a pressure of only $10 \mathrm{~atm}$ at $4.2 \mathrm{~K}$ is sufficient to reduce the range of the proton to $\sim 60 \mu \mathrm{m}$, whereas about 1000 atm would be needed at room temperature. Thus, the excellent position resolution of the detector is expected. Furthermore, the operation of the detector at a relatively low pressure simplifies the fabrication of the gas chamber, and the gas converter ensures good uniformity in detection efficiency at all pixels.

An InSb semiconductor detector ${ }^{3}$ was adopted as a solidstate detector. This type of detector is expected to have an energy resolution twice that of a silicon semiconductor detector. The InSb semiconductor is suitable for use at cryogenic temperatures since its electron and hole mobilities are about 40 and 1.5 times larger, respectively, than those of silicon. Moreover, arrays of InSb detectors should be developed in the near future since they are already commercially available as infrared detectors. Such an imaging neutron detector would be a powerful tool for ultrasmall-angle neutron scattering experiments where a detector with high position resolution and high counting rate is required.

In this article, we report on results obtained from the 


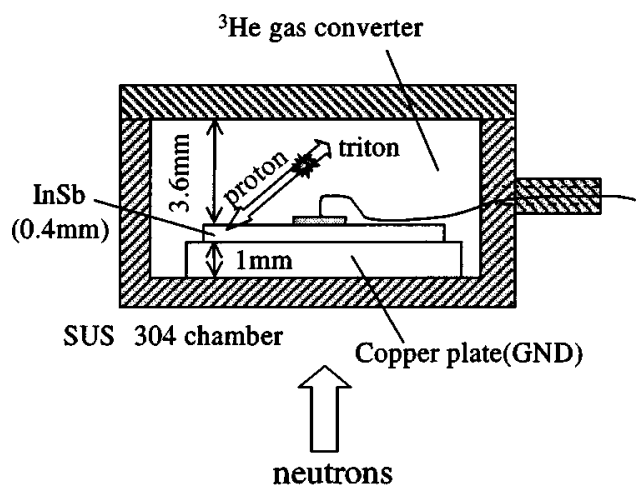

(a) side view

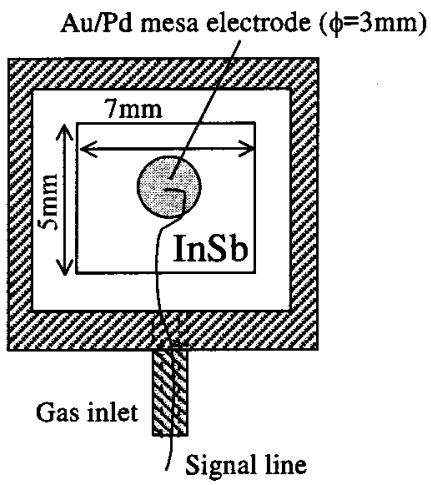

(b) top view

FIG. 1. Schematic views of the cryogenic neutron detector comprising an InSb semiconductor detector and a supercritical helium-3 gas converter. The detector operates at up to $1.5 \mathrm{~atm}$ at $4.2 \mathrm{~K}$.

neutron detector proposed above. We confirmed the operating principle of the detector equipped with a single InSb semiconductor detector. The detector successfully detected neutrons at $4.2 \mathrm{~K}$ in gas pressures up to $1.5 \mathrm{~atm}$, where the density of the helium-3 nucleus corresponds to that at $\sim 160$ $\mathrm{atm}$ at room temperature. Here we describe the structure, operation, and neutron-detection characteristics of the detector developed.

\section{CRYOGENIC NEUTRON DETECTOR}

The structure of the developed cryogenic neutron detector is shown in Fig. 1. The InSb semiconductor detector measures $5 \times 7 \mathrm{~mm}^{2}$ and is $0.4 \mathrm{~mm}$ thick, and is attached to a 1-mm-thick copper plate by indium solder. Details of the fabrication of the detector and the basic characteristics of alpha detection can be found elsewhere. ${ }^{4}$ The InSb semiconductor detector was fixed inside the gas chamber by STYCAST. The chamber is made of SUS 304 and can withstand pressure up to $15 \mathrm{~atm}$. The neutron-absorption space is about $3.6 \mathrm{~mm}$ deep, which is set by the geometrical gap between the InSb semiconductor detector and the window of the gas chamber. Helium-3 gas was introduced into the chamber through a SUS tube with an inner diameter of $1 \mathrm{~mm}$, and the gas pressure was adjusted from 0 to $1.5 \mathrm{~atm}$ by the outer bulb and a regulator. The chamber was immersed in liquid helium-4 in order to cool the InSb detector and the introduced helium- 3 conversion gas to $4.2 \mathrm{~K}$ (at this temperature the helium-3 stays in gas phase since its boiling point is 3.19

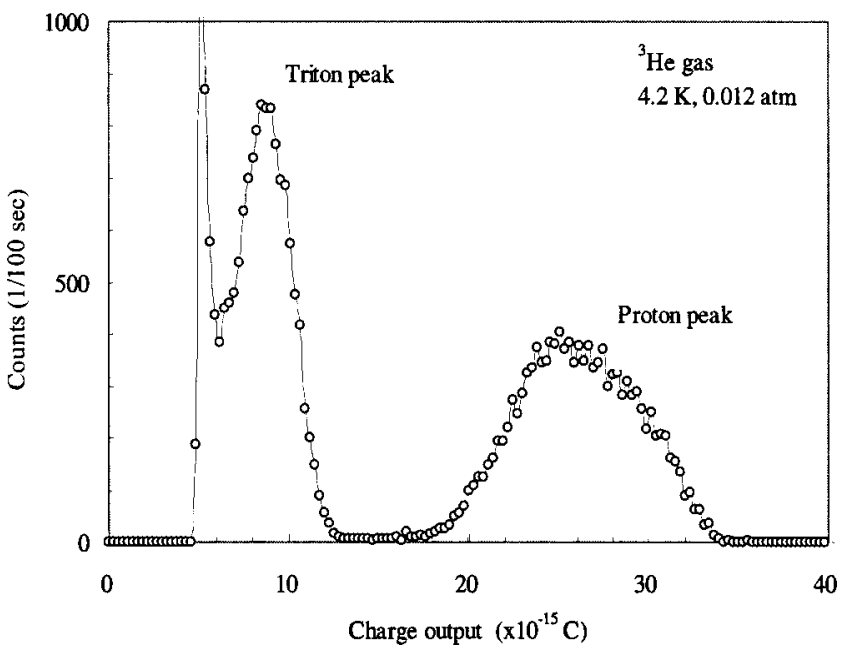

FIG. 2. Pulse height distribution obtained by the InSb detector operated at $4.2 \mathrm{~K}$. Helium-3 gas was filled at $0.012 \mathrm{~atm}$.

$\mathrm{K})$. At pressures above $1.15 \mathrm{~atm}$, helium- 3 is supercritical with no clear separation of gas and liquid. The density of helium-3 at $1.5 \mathrm{~atm}$ and $4.2 \mathrm{~K}$ was estimated as 0.0194 $\mathrm{g} \mathrm{cm}^{-3}$ by solving the equation of state with empirical virial constants, ${ }^{5}$ where the density corresponds to that at $160 \mathrm{~atm}$ at room temperature.

The basic characteristics of the neutron detector were investigated using thermal neutrons in the T-1 beam line, JRR-3M, at the Japan Atomic Energy Research Institute. Neutrons enter the detector from the back of the InSb semiconductor and are absorbed in helium-3. Secondary particles-a $574 \mathrm{keV}$ proton and a $191 \mathrm{keV}$ triton-created during the ${ }^{3} \mathrm{He}(n, p)$ T reaction are emitted in opposite directions. Neutrons are counted by the detection of either a proton or a triton by the InSb detector which is placed in a backward direction relative to incident neutrons. The counting rate of the InSb detector was set at less than $1000 \mathrm{cps}$ by collimating the neutron beam.

The signal line of the detector was taken outside the high-pressure chamber and connected to a preamplifier (model 2003BT, Canberra) operating at room temperature. The distance between the detector and the preamplifier was about $1 \mathrm{~m}$. The signal pulses were amplified and shaped into near Gaussians at the spectroscopy amplifier (Canberra, 2021) with a shaping time of $1.5 \mu \mathrm{s}$, and the pulse heights were analyzed by a multichannel analyzer. Signal pulses at the preamplifier outputs were viewed on an oscilloscope.

\section{EXPERIMENTS}

\section{A. Neutron detection at $4.2 \mathrm{~K}$ with low gas pressure}

We first checked the operation of the InSb semiconductor detector at $4.2 \mathrm{~K}$ with dilute helium-3 gas. Figure 2 shows the pulse height distribution obtained at a gas pressure of $0.012 \mathrm{~atm}$. The two peaks are clearly evident: their height ratio is $2.98 \pm 0.01$ (mean $\pm \mathrm{SD}$ ) and the number of counts under each peak was almost the same. These peaks can be attributed to protons and tritons created in the ${ }^{3} \mathrm{He}(n, p) \mathrm{T}$ reaction. 


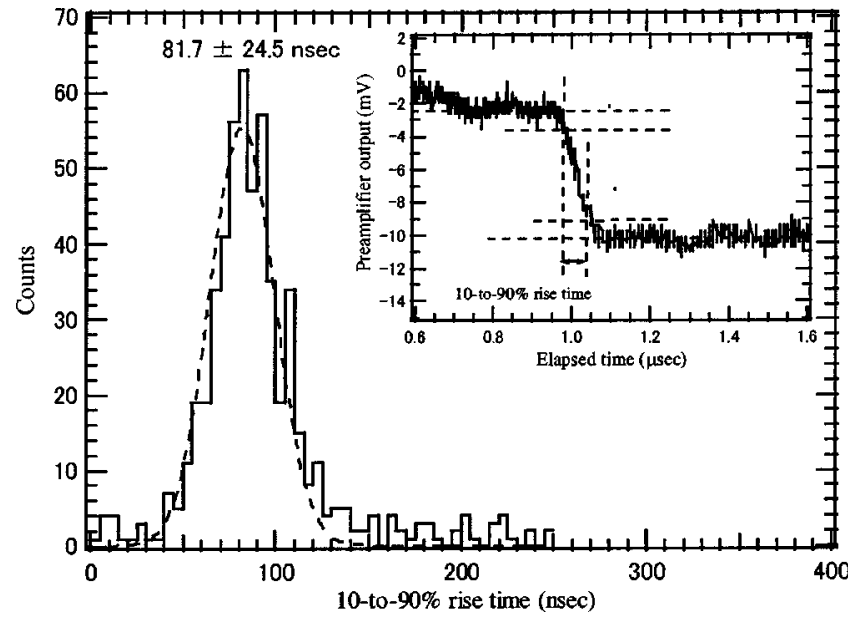

FIG. 3. Rise-time (10\%-90\%) distribution of neutron signal pulses from the InSb detector at $4.2 \mathrm{~K}$ and $0.012 \mathrm{~atm}$. Inset shows one of the preamplifier outputs. The rise time of the signals was $81.7 \pm 24.5 \mathrm{~ns}$.

Figure 3 presents analyzed results of the 10\%-90\% rise time of the preamplifier outputs. One of the typical pulses is shown in the figure inset. The rise time of the preamplifier output was $81.7 \pm 24.5 \mathrm{~ns}$. The pulse shape and rise time were hardly altered when the gas pressure was increased to $1.5 \mathrm{~atm}$.

\section{B. Pulse height distribution and detection efficiency}

We evaluated the pulse height distribution at gas pressures of $0.012-1.51 \mathrm{~atm}$ at $4.2 \mathrm{~K}$. Figures 4(a) and 4(b) show the pulse height distributions at gas pressures of 0.012-0.16 and $0.16-1.51 \mathrm{~atm}$, respectively. Double peaks are particularly evident in Fig. 4(a), with the width of the higher peak decreasing and the shape degrading as the gas pressure increases. The width decreased up to $\sim 0.1 \mathrm{~atm}$. At pressures above $0.16 \mathrm{~atm}$, the total sum counts reduced while the pulse height distribution remained approximately constant, as shown in Fig. 4(b). The shape of the smaller peak was virtually the same at all gas pressures.

Figure 5 shows the peak channel of the larger pulse and the sum counts (in channel 46 and above) in Fig. 4 as a function of the filled gas pressure. At the peak position, the reduction in the pulse height stopped at $\sim 0.1 \mathrm{~atm}$ with nearly the same pulse height being sustained at higher gas pressures. On the other hand, the sum counts corresponding to detection efficiency increased with pressures up to a maximum of $0.12 \mathrm{~atm}$, and then decreased with the gas pressure. The sum counts were reduced by about half at $1.5 \mathrm{~atm}$.

\section{DISCUSSION}

\section{A. Characteristics of the InSb detector at $4.2 \mathrm{~K}$}

\section{Identification of the peaks}

Here we discuss the identification of the two peaks observed in the pulse height of the InSb detector. The InSb detector is sensitive to both the particle energy and stopping power, depending on the thickness of the depletion layer and the range of the particle in InSb. We did not know which mode of operation the detector was in since the depletion

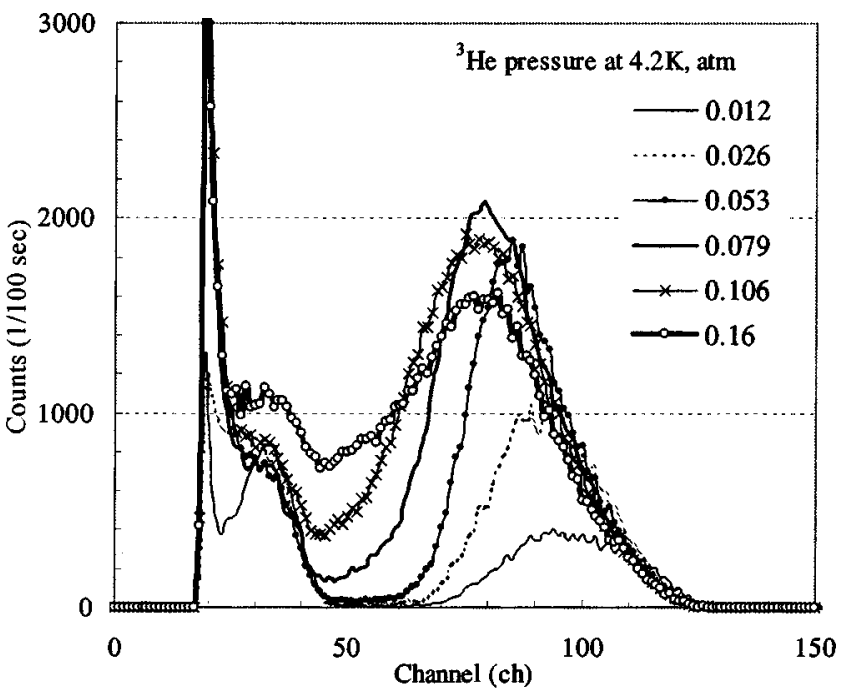

(a) $0.012 \sim 0.16 \mathrm{~atm}$

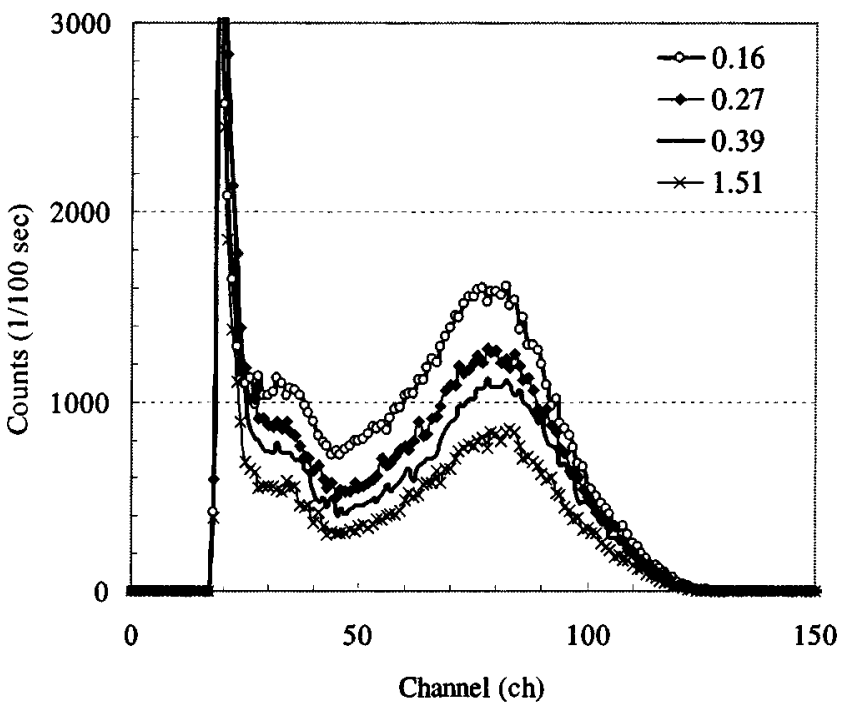

(b) $0.16 \sim 1.51 \mathrm{~atm}$

FIG. 4. Pulse height distributions of the InSb detector at gas pressures of $0.012-1.51 \mathrm{~atm}$ at $4.2 \mathrm{~K}$.

layer of the InSb detector was unknown at this stage. The detector operates as stopping-power sensitive when the range of the secondary particle is shorter than the thickness of the depletion layer, and as energy sensitive in the opposite case. In the stopping-power mode, the ratio of the stopping powers of protons to tritons should determine the ratio of the peak channels in the pulse height distribution. The calculated ratio of the stopping power between protons and tritons is $1.4-1.6,{ }^{6}$ which is about half the ratio evident in Fig. 2. On the other hand, the ratio of the initial energy of the particles, i.e., $E_{\text {proton }} / E_{\text {triton }}=3.0(574 / 191 \mathrm{keV})$, should be observed in the energy-sensitive mode. This agreed well with the measured ratio, and hence we concluded that the InSb detector was operating in energy-sensitive mode, where the larger and smaller peaks in Figs. 2 and 4 correspond to protons and tritons, respectively. From the peak value of charge output by protons, the effective energy $\epsilon_{\text {eff }}$ to create an electron-hole pair in the InSb was estimated as $3.6 \mathrm{eV}$. This value was about 1 order of magnitude larger than that simply estimated 


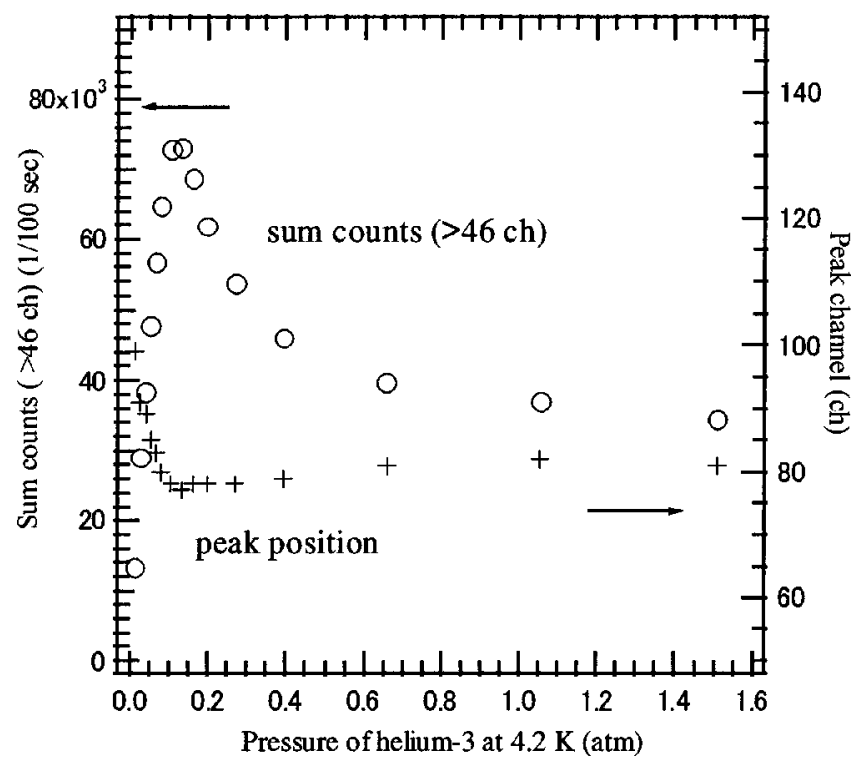

FIG. 5. Sum counts and peak channel of the proton peak vs helium-3 pressure at $4.2 \mathrm{~K}$.

from the energy gap of the InSb, inferring significant imperfection in the charge collection by the InSb detector.

\section{Estimating the thickness of the depletion layer}

The depletion layer of InSb should be larger than the range of a proton in the InSb since the InSb detected the full energy of the proton. The ranges of a proton and a triton in InSb $\left(\rho=5.78 \mathrm{~g} \mathrm{~cm}^{-3}\right)$ were estimated at 5.8 and $1.7 \mu \mathrm{m}$, respectively. ${ }^{6}$ On the other hand, the InSb detector could not measure the full energy of alpha particles, which have a calibrated energy of $3.91 \mathrm{MeV}$ from our ${ }^{241} \mathrm{Am}$ source with a thin gold coating film. ${ }^{4}$ The energy deposited by the alpha particles was estimated to be $\sim 1.6 \mathrm{MeV}$ from the measured value of $\epsilon_{\text {eff }}$. From this result, we determined the thickness of the depletion layer in the InSb detector as $\sim 6.5 \mu \mathrm{m}$ from the range of the alpha particles deposited in the InSb. For the case of neutron detection, the depletion layer should not be too large since this increases the absorption of gamma rays and $\mathrm{x}$ rays, and hence the background signal. From these considerations, we found that when it was operated at near zero bias voltage, the depletion thickness of the InSb detector was optimal for the detection of neutrons (protons).

\section{Time response of the detector}

The charge collection time in the InSb detector is estimated from the following formula:

$$
t \sim d^{2} / \mu V_{b},
$$

where $d$ is the thickness of the depletion layer, $\mu$ is the carrier mobility of carrier, and $V_{b}$ is the bias voltage. In our $\mathrm{InSb}$ detector, the charge collection time becomes ideally $\sim 0.01$ ns (taking the mobility of electrons $\mu_{e}$ as 78000 $\mathrm{cm}^{2} \mathrm{~V}^{-1} \mathrm{~s}^{-1}$ and the inherent bias voltage $V_{b}$ as $\left.0.68 \mathrm{~V}\right) .^{7}$ The fast rise time of the preamplifier output of $\sim 10 \mathrm{~ns}$ was expected from the time response of the electronic pulser. However, although the observed rise time was slower than this, the measured charge-collection time was about 1 order of magnitude better than that reported previously. ${ }^{7}$ Two reasons for this are: (i) imperfect charge collection due to charge trapping in the InSb and (ii) a reduction in the inherent electric field in the depletion layer due to the presence of a plasma column.

We applied a bias voltage of $\pm 0.05 \mathrm{~V}$ to the InSb detector to improve the charge collection. However, this caused large fluctuations in the baseline preamplifier output and negligible improvement in charge collection; this is attributable to the low resistance of the InSb detector, which near a zero bias voltage is $1.5 \mathrm{k} \Omega$ at $4.2 \mathrm{~K}$. Therefore, changes to the $\mathrm{InSb}$ detector, such as in the detector structure, purification of the material, and better crystal growth, are needed to improve the time response and charge collection characteristics.

\section{B. Neutron detection characteristics of the helium-3 gas converter}

\section{Pulse height dependence on gas pressure}

The peak position in the pulse height distribution was related to the applied gas pressure, as shown in Fig. 5. This dependence on gas pressure can be understood qualitatively by taking account of the averaged energy loss $\delta E$ of a proton in the gas. The peak position in the pulse height distribution should be proportional to $E-\delta E$, where $E$ is the initial energy of a proton. The averaged energy loss of a secondary particle in the gas is

$$
\delta E \propto \sum_{i}(d E / d x)_{i} \Delta x_{i},
$$

where $(d E / d x)_{i}$ is the stopping power of a proton in the gas, and $\Delta x_{i}$ is a distance component equal to the range of a proton divided by an integer $n$. The summation on the righthand side is carried out along the track until the proton reaches the $\mathrm{InSb}$ detector.

We now consider the low-pressure case where the range of the proton is much longer than the depth of the neutron absorption space of the detector. In this case, the energy loss of the proton in the gas, $\delta E$, should be negligibly small for most neutron events. Increasing the gas pressure increases the energy loss in the gas, and this should decrease the peak position $(\propto E-\delta E)$. On the other hand, at a much higher pressure where the range of a proton is shorter than the depth of the neutron absorption space, the energy loss of a proton in the gas should be constant regardless of the gas pressure since the dependence of the stopping power and the track on the gas pressure cancel out; i.e., $(d E / d x)_{i} \propto p$ and $\Delta x_{i}$ $\sim 1 / p$. Hence, the reduction in the peak channel should not be observed at a sufficiently high gas pressure. Actually, the measured peak channel was reduced by up to $0.1 \mathrm{~atm}$ and nearly the same peak channel was maintained at a higher pressure. Therefore, the above explanation seems plausible.

The pulse height distribution remains the same even at high pressure. This feature enables discrimination of neutron signals from the background by simply setting a constant pulse height level at a particular filling gas pressure. This is a very useful characteristic for a neutron detector in which neutron signals always need to be discriminated from the background. 


\section{Detection efficiency at high pressure}

The number of counts detected by the InSb detector was strongly correlated with the filling gas pressure, as shown in Fig. 5. The change in sum counts could not be attributed to counting loss of signals with low pulse heights since the pulse height distribution hardly changed above 0.1 atm, as shown in Fig. 4(b). If we assume that the range of the proton was inversely proportional to the density, following the Bethe theory, the detection efficiency of the proton should be constant at moderate and higher gas pressures. This was confirmed by a preliminary Monte Carlo calculation of detection efficiency. The reason for the significant decrease in measured detection efficiency is not clear at this stage, but it may be explained by a greater reduction in the range of protons in the high-density, low-temperature helium gas. This should be clarified by future investigations of the stopping power of low-temperature helium-3 gas.

\section{ACKNOWLEDGMENTS}

The authors are indebted to $\mathrm{H}$. Yamagishi, Dr. S. Masaoka, Dr. K. Aizawa, and Dr. K. Soyama for fruitful discussions and technical assistance.

${ }^{1}$ S. Kishimoto, Y. Isozumi, R. Katano, and H. Takekoshi, Nucl. Instrum. Methods Phys. Res. A 262, 413 (1987).

${ }^{2}$ C. A. Baker et al., Nucl. Instrum. Methods Phys. Res. A 487, 511 (2002).

${ }^{3}$ I. Kanno, F. Yoshihara, R. Nouchi, O. Sugiura, T. Nakamura, and M. Katagiri, Rev. Sci. Instrum. 73, 2533 (2002).

${ }^{4}$ S. Hishiki, I. Kanno, R. Nouchi, O. Sugiura, T. Nakamura, and M. Katagiri (unpublished).

${ }^{5}$ T. R. Roberts, R. H. Sherman, and S. G. Sydoriak, J. Res. Natl. Bur. Stand., Sect. A 68, 567 (1964).

${ }^{6}$ J. F. Ziegler, J. P. Biersack, and U. Littmark, The Stopping and Range of Ions in Matter (Pergamon, New York, 1985).

${ }^{7}$ I. Kanno, F. Yoshihara, R. Nouchi, O. Sugiura, T. Nakamura, and M. Katagiri, Rev. Sci. Instrum. 74, 3968 (2003). 